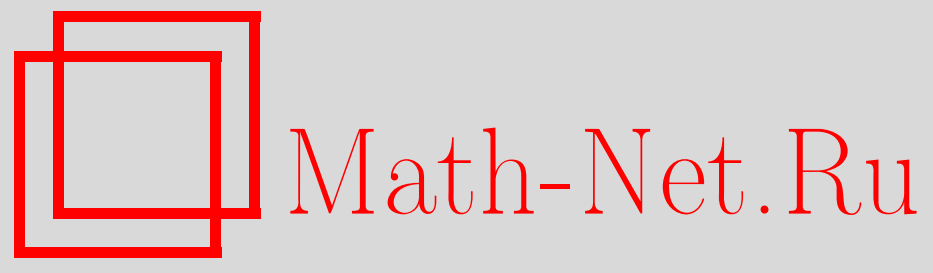

В. А. Кудинов, Р. М. Клеблеев, Е. А. Куклова, Получение точных аналитических решений нестационарных задач теплопроводности ортогональными методами, Вестн. Сам. гос. техн. ун-та. Сер. Физ.-мат. науки, 2017, номер 1, 197-206

DOI: https://doi.org/10.14498/vsgtu1521

Использование Общероссийского математического портала MathNet.Ru подразумевает, что вы прочитали и согласны с пользовательским соглашением

http://www.mathnet.ru/rus/agreement

Параметры загрузки:

IP : 3.85 .5 .30

26 апреля 2023 г., 09:15:28

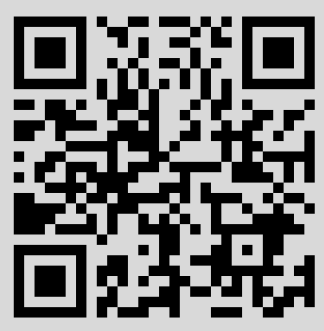


Вестн. Сам. гос. техн. ун-та. Сер. Физ.-мат. науки. 2017. Т. 21, № 1. С. $197-206$ ISSN: 2310-7081 (online), 1991-8615 (print) doi) http://doi.org/10.14498/vsgtu1521

УДК 517.958:[536.2+539.219.3]

\title{
Получение точных аналитических решений нестационарных задач теплопроводности ортогональными методами
}

\author{
В. А. Кудинов, Р. М. Клеблеев, Е. А. Куклова
}

Самарский государственный технический университет,

Россия, 443100, Самара, ул. Молодогвардейская, 244.

\section{Аннотация}

При совместном использовании ортогональных методов Л. В. Канторовича, Бубнова - Галеркина и интегрального метода теплового баланса получено точное аналитическое решение нестационарной задачи теплопроводности для бесконечной пластины при симметричных граничных условиях первого рода. Нахождение точного решения при использовании приближенных методов оказалось возможным вследствие использования тригонометрических координатных функций, обладающих свойством ортогональности. Их применение позволяет находить собственные числа не через решение краевой задачи Штурма-Лиувилля, в котором интегрированию подлежит дифференциальное уравнение второго порядка, а через решение дифференциального уравнения относительно неизвестной функций времени, являющегося уравнением первого порядка. Благодаря этому же свойству координатных функций при нахождении из начальных условий констант интегрирования удается избежать решения больших систем алгебраических линейных уравнений с плохо обусловленными матрицами коэффициентов. В связи с чем значительно упрощается как процесс получения решения, так и окончательная формула для него при возможности нахождения не только приближенного, но и точного аналитического решения в форме бесконечного ряда.

Ключевые слова: нестационарная теплопроводность, метод Л. В. Канторовича, метод Бубнова-Галеркина, интегральный метод теплового баланса, тригонометрические координатные функции, точное аналитическое решение, ортогональность координатных функций.

Получение: 28 октября 2016 г. / Исправление: 11 февраля 2017 г. Принятие: 13 марта 2017 г. / Публикация онлайн: 19 марта 2017 г.

\section{Краткое сообщение}

(웅 Контент публикуется на условиях лицензии Creative Commons Attribution 4.0 International (https://creativecommons.org/licenses/by/4.0/deed.ru)

\section{Образец для цитирования}

Кудинов В. А., Клеблеев Р. М., Куклова Е. А. Получение точных аналитических решений нестационарных задач теплопроводности ортогональными методами // Вестн. Сам. гос. техн. ун-та. Сер. Физ.-мат. науки, 2017. Т. 21, № 1. С. 197-206. doi: $10.14498 /$ vsgtu1521.

\section{Сведения об авторах}

Василий Александрович Кудинов (10) http://orcid.org/0000-0002-3071-5168

доктор физико-математических наук, профессор; заведующий кафедрой; каф. теоретических основ теплотехники и гидродинамики; e-mail: totig@yandex.ru

Руслан Мухтарович Клеблеев (D) http://orcid.org/0000-0002-6944-8503

магистрант; каф. теоретических основ теплотехники и гидродинамики;

e-mail: uio11230list.ru 
При получении приближенных аналитических решений большое распространение получили ортогональные методы Л. В. Канторовича, БубноваГалеркина и интегральный метод теплового баланса, важное преимущество которых перед классическими аналитическими методами (Фурье, интегральных преобразований, функций Грина и др.) состоит в том, что при их использовании на вид дифференциального оператора краевой задачи не накладывается каких-либо ограничений $[1,2]$. И, в частности, они могут быть применены к интегрированию дифференциальных уравнений, не допускающих разделения переменных [3,4], например, нелинейных, с переменными физическими свойствами среды и др. Решение находится в виде степенных (или тригонометрических) полиномов по координатам текущей точки с экспоненциально стабилизирующимися во времени коэффициентами. В результате применения этих методов для линейных и нелинейных краевых задач можно получить простые по форме аналитические решения, в том числе и точные (для линейных задач), содержащие все основные физические свойства среды в явном виде.

В настоящей работе показывается, как на основе ортогональных методов Л. В. Канторовича, Бубнова-Галеркина и интегрального метода теплового баланса можно получать не только приближенные, но и точные аналитические решения задач нестационарной теплопроводности. Рассмотрим идею метода на примере решения краевой задачи для бесконечной пластины толщиной $\delta$ в следующей постановке:

$$
\begin{gathered}
\frac{\partial T(x, t)}{\partial t}=a \frac{\partial^{2} T(x, t)}{\partial x^{2}}, \quad t>0, \quad 0<x<\delta \\
T(x, 0)=T_{0}, \quad \partial T(0, t) / \partial x=0, \quad T(\delta, t)=T_{\text {ст }} .
\end{gathered}
$$

Здесь $T$ - температура, $x$ - координата, $t$ - время, $T_{\text {ст }}$ - температура на стенках пластины, $T_{0}$ - начальная температура, $a$ - коэффициент температуропроводности.

Для приведения задачи (1), (2) к безразмерному виду введем следующие обозначения:

$$
\Theta=\left(T-T_{\text {ст }}\right) /\left(T_{0}-T_{\text {ст }}\right), \quad \text { Fo }=a t / \delta^{2}, \quad \xi=x / \delta,
$$

где $\Theta$, Fo, $\xi$ - соответственно, безразмерные температура, время и координата. Задача (1), (2) в обозначениях (3) принимает вид

$$
\begin{gathered}
\frac{\partial \Theta(\xi, \text { Fo })}{\partial F_{\circ}}=\frac{\partial^{2} \Theta(\xi, \text { Fo })}{\partial \xi^{2}}, \quad \text { Fo }>0, \quad 0<\xi<1 ; \\
\Theta(\xi, 0)=1 ; \\
\frac{\partial \Theta(0, \text { Fo })}{\partial \xi}=0, \quad \Theta(1, \text { Fo })=0 .
\end{gathered}
$$

Екатерина Александровна Куклова (D) http://orcid.org/0000-0002-4836-5565 магистрант; каф. теоретических основ теплотехники и гидродинамики; e-mail: kyklova_1993@mail.ru 
Следуя методу Л. В. Канторовича, решение задачи (4)-(6) ищется в виде

$$
\Theta(\xi, \mathrm{Fo})=\sum_{k=1}^{n} f_{k}(\mathrm{Fo}) \varphi_{k}(\xi)
$$

где здесь и далее $f_{k}(\mathrm{Fo})$ - неизвестные функции времени; $\varphi_{k}(\xi)-$ координатные функции.

В работах $[3,4]$ в качестве координатных принимаются алгебраические полиномы. Так как алгебраические координатные функции не обладают свойством ортогональности, то при их использовании могут быть получены лишь приближенные аналитические решения. Найдем решение задачи (4)-(6) при помощи тригонометрических координатных функций вида

$$
\varphi_{k}(\xi)=\cos \left(\frac{(2 k-1) \pi}{2} \xi\right)
$$

Очевидно, что (7) с учетом (8) удовлетворяет граничным условиям (6). Потребуем, чтобы уравнение (4) на отрезке пространственной переменной $(0 \leqslant \xi \leqslant 1)$ выполнялось в среднем, то есть потребуем выполнения следующего интеграла теплового баланса:

$$
\int_{0}^{1} \frac{\partial \Theta(\xi, \mathrm{Fo})}{\partial \mathrm{Fo}_{\mathrm{o}}} d \xi=\int_{0}^{1} \frac{\partial^{2} \Theta(\xi, \mathrm{Fo})}{\partial \xi^{2}} d \xi
$$

Для нахождения решения задачи (4)-(6) в первом приближении подставим (7), ограничиваясь одним членом ряда, в (9):

$$
\int_{0}^{1} \frac{\partial}{\partial \mathrm{Fo}}\left[f_{1}(\mathrm{Fo}) \cos \left(\frac{\pi}{2} \xi\right)\right] d \xi-\int_{0}^{1} \frac{\partial^{2}}{\partial \xi^{2}}\left[f_{1}(\mathrm{Fo}) \cos \left(\frac{\pi}{2} \xi\right)\right] d \xi=0
$$

Соотношение (10) относительно неизвестной функции $f_{1}(\mathrm{Fo})$ приводится к обыкновенному дифференциальному уравнению

$$
\frac{d f_{1}(\mathrm{Fo})}{d \mathrm{Fo}_{\mathrm{o}}}+\frac{\pi^{2}}{4} f_{1}(\mathrm{Fo})=0
$$

после интегрирования которого находим

$$
f_{1}(\mathrm{Fo})=C_{1} \exp \left(-\frac{\pi^{2}}{4} \mathrm{Fo}\right)
$$

где $C_{1}$ - постоянная интегрирования. Подставляя $(8),(11)$ в $(7)$, находим

$$
\Theta(\xi, \text { Fo })=C_{1} \exp \left(-\frac{\pi^{2}}{4} \mathrm{Fo}\right) \cos \left(\frac{\pi}{2} \xi\right)
$$

Для определения постоянной интегрирования $C_{1}$ составим невязку начального условия (5) и потребуем ортогональности невязки к координатной функции $\varphi_{1}(\xi)$ :

$$
\int_{0}^{1}\left[C_{1} \cos \left(\frac{\pi}{2} \xi\right)-1\right] \cos \left(\frac{\pi}{2} \xi\right) d \xi=0 .
$$


Определяя интегралы в (13), относительно постоянной $C_{1}$ получаем алгебраическое линейное уравнение, из решения которого находится $C_{1}=4 / \pi$.

Соотношение (12) с учетом найденного значения постоянной интегрирования принимает вид

$$
\Theta(\xi, \mathrm{Fo})=\frac{4}{\pi} \exp \left(-\mu_{1} \mathrm{Fo}\right) \cos \left(\frac{\pi}{2} \xi\right)
$$

где $\mu_{1}=\pi^{2} / 4$. Отметим, что $\mu_{1}$ совпадает с первым собственным числом краевой задачи Штурма-Лиувилля при решении задачи (4)-(6) классическими аналитическими методами [5].

Соотношение (14) представляет решение задачи (4)-(6) в первом приближении. Оно точно удовлетворяет уравнению (4) и граничным условиям (6) и в первом приближении - начальному условию (5). Результаты расчетов по формуле (14) в сравнении с точным решением [5] приведены на рис. 1. Анализ численных данных позволяет заключить, что для Fo $\geqslant 0.1$ расхождение результатов не превышает $5 \%$.

Для решения задачи (4)-(6) во втором приближении составим невязку уравнения (4) и потребуем ортогональности невязки к координатным функциям $\varphi_{1}(\xi)$ и $\varphi_{2}(\xi)$ (реализация ортогонального метода Бубнова-Галеркина):

$$
\begin{aligned}
& \int_{0}^{1}\left[\frac{\partial \Theta(\xi, \mathrm{Fo})}{\partial \mathrm{Fo}}-\frac{\partial^{2} \Theta(\xi, \mathrm{Fo})}{\partial \xi^{2}}\right] \varphi_{1}(\xi) d \xi=0 \\
& \int_{0}^{1}\left[\frac{\partial \Theta(\xi, \mathrm{Fo})}{\partial \mathrm{Fo}}-\frac{\partial^{2} \Theta(\xi, \mathrm{Fo})}{\partial \xi^{2}}\right] \varphi_{2}(\xi) d \xi=0 .
\end{aligned}
$$

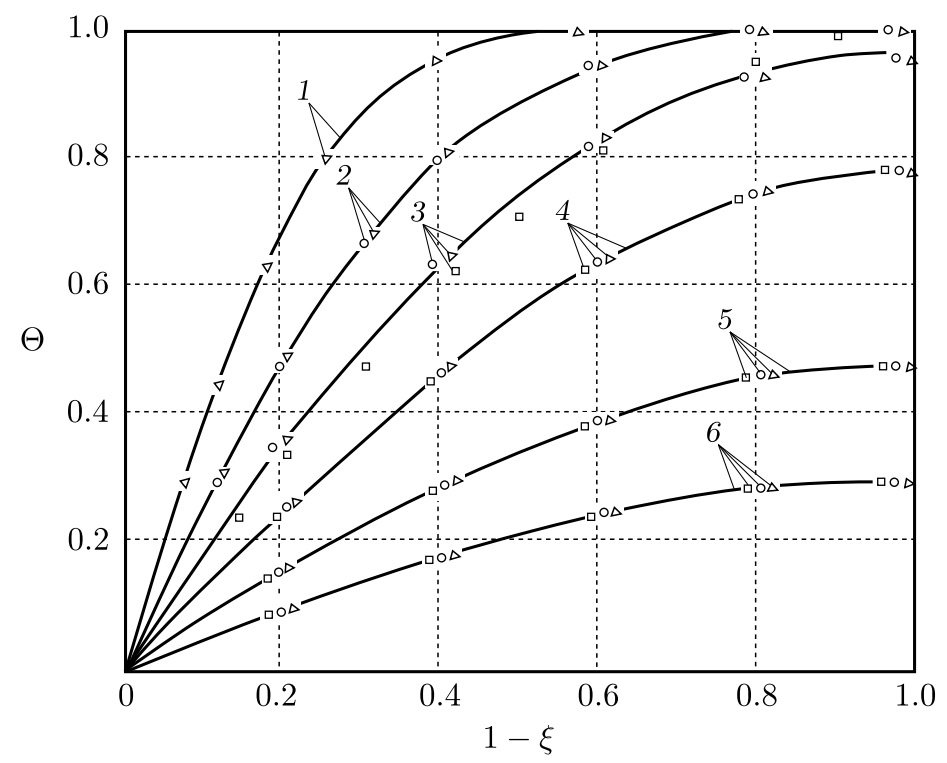

Рис. 1. Решение задачи (4)-(6) при различных Fo: сплошные линии - точное решение, $\square-$ первое приближение, о- второе приближение, $\triangle$ - третье приближение; $1-$ Fo $=0.02,2-$ $\mathrm{Fo}=0.05,3-\mathrm{Fo}=0.1,4-\mathrm{Fo}=0.2,5-$ Fo $=0.4,6-\mathrm{Fo}=0.6$

[Figure 1. The solution of the problem (4)-(6) for different Fo: solid lines - the exact solution, $\square-$ the first approximation, $\triangle-$ the second approximation, $\triangle-$ the third approximation; $1-\mathrm{Fo}=0.02,2-\mathrm{Fo}=0.05,3-\mathrm{Fo}=0.1,4-\mathrm{Fo}=0.2,5-\mathrm{Fo}=0.4,6-$ Fo $=0.6]$ 
Подставляя (7) (ограничиваясь двумя членами ряда) в (15), находим

$$
\begin{aligned}
& \int_{0}^{1}\left[f_{1}^{\prime}(\mathrm{Fo}) \varphi_{1}(\xi)+f_{2}^{\prime}(\mathrm{Fo}) \varphi_{2}(\xi)+f_{1}(\mathrm{Fo}) \varphi_{1}^{\prime \prime}(\xi)+f_{2}(\mathrm{Fo}) \varphi_{2}^{\prime \prime}(\xi)\right] \varphi_{1}(\xi) d \xi=0 \\
& \int_{0}^{1}\left[f_{1}^{\prime}(\mathrm{Fo}) \varphi_{1}(\xi)+f_{2}^{\prime}(\mathrm{Fo}) \varphi_{2}(\xi)+f_{1}(\mathrm{Fo}) \varphi_{1}^{\prime \prime}(\xi)+f_{2}(\mathrm{Fo}) \varphi_{2}^{\prime \prime}(\xi)\right] \varphi_{2}(\xi) d \xi=0
\end{aligned}
$$

где $f_{1}^{\prime}(\mathrm{Fo})=d f_{1}(\mathrm{Fo}) / d \mathrm{Fo}, f_{2}^{\prime}(\mathrm{Fo})=d f_{2}(\mathrm{Fo}) / d F_{o} ; \varphi_{1}^{\prime \prime}(\xi)=d^{2} \varphi_{1}(\xi) / d \xi^{2}, \varphi_{2}^{\prime \prime}(\xi)=$ $d^{2} \varphi_{2}(\xi) / d \xi^{2}$.

После определения интегралов соотношения (16) относительно неизвестных функций времени $f_{1}(\mathrm{Fo})$ и $f_{2}(\mathrm{Fo})$ в силу ортогональности косинусов получаем следующие раздельные обыкновенные дифференциальные уравнения относительно $f_{1}(\mathrm{Fo})$ и $f_{2}(\mathrm{Fo})$ :

$$
f_{1}^{\prime}(\mathrm{Fo})+\frac{\pi^{2}}{4} f_{1}(\mathrm{Fo})=0, \quad f_{2}^{\prime}(\mathrm{Fo})+\frac{9 \pi^{2}}{4} f_{2}(\mathrm{Fo})=0,
$$

которые легко интегрируются:

$$
f_{1}(\mathrm{Fo})=C_{1} \exp \left(-\frac{\pi^{2}}{4} \mathrm{Fo}\right), \quad f_{2}(\mathrm{Fo})=C_{2} \exp \left(-\frac{9 \pi^{2}}{4} \mathrm{Fo}\right),
$$

где $C_{1}, C_{2}$ - постоянные интегрирования.

Представляя (17) в (7), получаем

$$
\Theta(\xi, \mathrm{Fo})=C_{1} e^{-\frac{\pi^{2}}{4} \mathrm{Fo}} \cos \left(\frac{\pi}{2} \xi\right)+C_{2} e^{-\frac{9 \pi^{2}}{4} \mathrm{Fo}} \cos \left(\frac{3 \pi}{2} \xi\right) .
$$

Для определения постоянных интегрирования в (18) составим невязку начального условия (5) и потребуем ортогональности невязки к координатным функциям $\varphi_{1}(\xi)$ и $\varphi_{2}(\xi)$. Ввиду ортогональности косинусов неизвестные постоянные $C_{1}$ и $C_{2}$ в соотношениях для невязки разделятся:

$$
\begin{gathered}
\int_{0}^{1}\left[C_{1} \cos ^{2}\left(\frac{\pi}{2} \xi\right)-\cos \left(\frac{\pi}{2} \xi\right)\right] d \xi=0 \\
\int_{0}^{1}\left[C_{2} \cos ^{2}\left(\frac{3 \pi}{2} \xi\right)-\cos \left(\frac{3 \pi}{2} \xi\right)\right] d \xi=0 .
\end{gathered}
$$

Определяя интегралы в $(19),(20)$, находим $C_{1}=4 / \pi ; C_{2}=-4 /(3 \pi)$, с учетом которых соотношение (18) принимает вид

$$
\Theta(\xi, \text { Fo })=\frac{4}{\pi} e^{-\mu_{1} \mathrm{Fo}} \cos \left(\frac{\pi}{2} \xi\right)-\frac{4}{3 \pi} e^{-\mu_{2} \mathrm{Fo}} \cos \left(\frac{3 \pi}{2} \xi\right),
$$

где $\mu_{1}=\pi^{2} / 4 ; \mu_{2}=9 \pi^{2} / 4$.

Соотношение (21) представляет решение задачи (4)-(6) во втором приближении. Оно точно удовлетворяет уравнению (4) и граничным условиям (6) и приближенно (во втором приближении) - начальному условию (5). Отметим, что $\mu_{1}$ и $\mu_{2}$ совпадают с двумя первыми собственными числами, получаемыми при решении задачи (4)-(6) классическими аналитическими методами, а 
константы интегрирования $C_{1}$ и $C_{2}$ совпадают с двумя первыми коэффициентами, получаемыми из выполнения начального условия в точном аналитическом решении.

Результаты расчётов по формуле (21) в сравнении с точным аналитическим решением [5] приведены на рис. 1. Анализ численных данных позволяет заключить, что во втором приближении, по сравнению с первым, происходит существенное уточнение получаемого решения (при Fo $\geqslant 0.05$ оно практически совпадает с точным).

Найдем решение задачи (4)-(6) для любого приближения, то есть при $n \rightarrow \infty$. Составим невязку уравнения (4) и потребуем ортогональности невязки ко всем координатным функциям $\varphi_{k}(\xi)$ :

$$
\int_{0}^{1}\left[\sum_{k=1}^{\infty} \frac{d f_{k}(\mathrm{Fo})}{d \mathrm{Fo}} \varphi_{k}(\xi)+\mu_{k} \int_{0}^{1} \sum_{k=1}^{\infty} f_{k}(\mathrm{Fo}) \varphi_{k}(\xi)\right] \varphi_{j}(\xi) d \xi=0, j=1,2, \ldots
$$

где $\mu_{k}=(2 k-1)^{2} \pi^{2} / 4$. Соотношения (22) с учетом ортогональности косинусов приводятся к виду

$$
\frac{d f_{k}(\mathrm{Fo})}{d \mathrm{Fo}}+\mu_{k} f_{k}(\mathrm{Fo})=0, \quad k=1,2, \ldots
$$

После интегрирования (23) получаем

$$
f_{k}(\mathrm{Fo})=C_{k} \exp \left(-\mu_{k} \mathrm{Fo}\right), \quad k=1,2, \ldots,
$$

где $C_{k}$ - постоянные интегрирования. Подставим $(24)$ в $(7)($ при $n \rightarrow \infty)$ :

$$
\Theta(\xi, \mathrm{Fo})=\sum_{k=1}^{\infty} C_{k} \exp \left(-\mu_{k} \mathrm{Fo}\right) \varphi_{k}(\xi)
$$

Для определения постоянных интегрирования $C_{k}$ составим невязку начального условия (5) и потребуем ортогональности невязки ко всем координатным функциям $\varphi_{k}(\xi)$ :

$$
\int_{0}^{1}\left[\sum_{k=1}^{\infty} C_{k} \varphi_{k}(\xi)-1\right] \varphi_{j}(\xi) d \xi=0, \quad j=1,2, \ldots
$$

Последние соотношения ввиду ортогональности координатных функций (8) принимают вид

$$
\int_{0}^{1}\left[C_{k} \varphi_{k}^{2}(\xi)-\varphi_{k}(\xi)\right] d \xi=0, \quad k=1,2, \ldots
$$

Определяя интегралы в (26), находим $C_{k}$ :

$$
C_{k}=(-1)^{k+1} \frac{2}{\sqrt{\mu_{k}}}, \quad k=1,2, \ldots
$$


Подставляя найденные $C_{k}$ в $(25)$, получаем формулу

$$
\Theta(\xi, \mathrm{Fo})=\sum_{k=1}^{\infty}(-1)^{k+1} \frac{2}{\sqrt{\mu_{k}}} \exp \left(-\mu_{k} \mathrm{Fo}\right) \varphi_{k}(\xi),
$$

которая представляет собой точное аналитическое решение задачи (4)-(6).

На рис. 2 в качестве иллюстрации предложенного метода приведено точное решение (сплошные линии) задачи (4)-(6) и решение, полученное по формуле (25), когда используется 100 членов ряда, при различных значениях Fo.

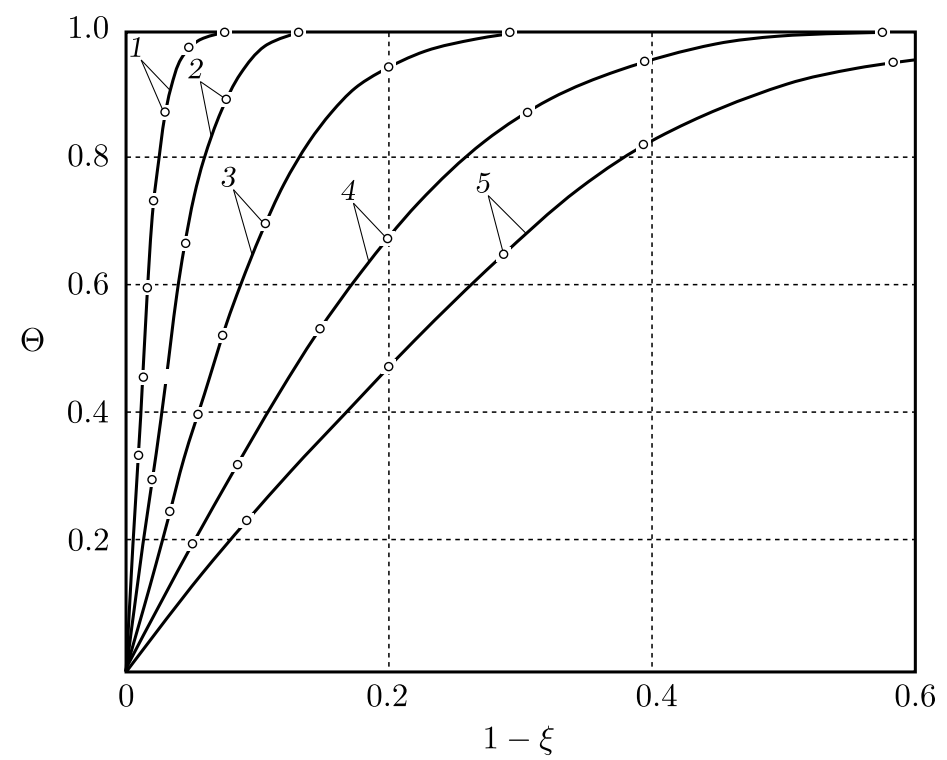

Рис. 2. Точное решение (сплошные линии) задачи (4)-(6) и решение, полученное по формуле (25), когда используется 100 членов ряда, при различных значения Fo: $1-$ Fo $=0.0001$, $2-\mathrm{Fo}=0.001,3-\mathrm{Fo}=0.0005,4-\mathrm{Fo}=0.02,5-\mathrm{Fo}=0.6$

[Figure 2. The exact solution (solid lines) of the problem (4)-(6) and the solution obtained by the formula (25), when 100 members of the series are used for different Fo: $1-$ Fo $=0.0001$,

$$
2-\mathrm{Fo}=0.001,3-\mathrm{Fo}=0.0005,4-\mathrm{Fo}=0.02,5-\text { Fo }=0.6]
$$

Выводы. На основе ортогональных методов Л. В. Канторовича, Бубнова-Галеркина и интегрального метода теплового баланса при использовании тригонометрических координатных функций получено точное аналитическое решение задачи теплопроводности для бесконечной пластины при симметричных граничных условиях первого рода. Благодаря ортогональности тригонометрических функций неизвестные функции времени в системе обыкновенных дифференциальных уравнений разделяются - в каждое уравнение входит лишь одна неизвестная величина. В итоге удается получить решение общего вида для неизвестных функций времени при любом числе приближений. Благодаря этому же свойству координатных функций при определении из начального условия констант интегрирования удается избежать решения больших систем алгебраических линейных уравнений с плохо обусловленными матрицами коэффициентов и получить общую формулу для нахождения этих констант. 
Преимущества такого метода получения аналитического решения в том, что при совместном использовании указанных методов на вид дифференциального уравнения краевой задачи практически не накладывается каких-либо ограничений. Причем собственные числа определяются из решения дифференциального уравнения относительно неизвестных функций времени (уравнение первого порядка), а не из краевой задачи Штурма-Лиувилля (уравнение второго порядка), решение которой требуется находить при использовании классических точных аналитических методов.

Ортогональные методы с тригонометрическими координатными функциями могут быть использованы и при решении краевых задач, дифференциальные уравнения которых не допускают разделения переменных (нелинейных, с переменными физическими свойствами среды и др.). Однако применительно к ним могут быть получены лишь приближенные аналитические решения. Трудности получения точных решений в форме бесконечных рядов связаны с тем, что для нелинейных краевых задач принцип линейной суперпозиции частных решений неприменим.

Конкурирующие интересы. Мы не имеем конкурирующих интересов.

Авторский вклад и ответственность. Все авторы принимали участие в разработке концепции статьи и в написании рукописи. Авторы несут полную ответственность за предоставление окончательной рукописи в печать. Окончательная версия рукописи была одобрена всеми авторами.

Финансирование. Работа выполнена при финансовой поддержке Министерства образования и науки РФ в рамках базовой части государственного задания ФГБОУ ВО «СамГТУ» (проект № 1.5551.2017/8.9).

\section{Библиографический список}

1. Канторович Л. В., Крылов В. И. Приближенные методы высшего анализа. Л.: Физматгиз, 1962. 708 с.

2. Цой П. В. Методы расчета отделъных задач тепломассопереноса. М.: Энергия, 1971. $382 \mathrm{c.}$

3. Кудинов В. А., Карташов Э. М., Калашников В. В. Аналитические решения задач тепломассопереноса и термоупругости для многослойных конструкиий. М.: Высшая школа, 2005. 430 с.

4. Кудинов В. А., Аверин Б. В., Стефанюк Е. В. Теплопроводность и термоупругость в многослойных конструкциях. М.: Высшая школа, 2008. 305 с.

5. Лыков А. В. Теория теплопроводности. М.: Высшая школа, 1967. 600 с. 
MSC: 35K05, 80A20, 35C10

\title{
Obtaining exact analytical solutions for nonstationary heat conduction problems using orthogonal methods
}

\author{
V. A. Kudinov, R. M. Klebleev, E. A. Kuklova
}

Samara State Technical University,

244, Molodogvardeyskaya st., Samara, 443100, Russian Federation.

\begin{abstract}
Through the interplay of orthogonal methods by L. V. Kantorovich, Bubnov-Galerkin and a heat balance integral method there have been obtained an exact analytical solution of a nonstationary heat conduction problem for an infinite plate under the symmetrical first-type boundary conditions. It was possible to obtain an exact solution through the employment of approximate methods due to the appliance of trigonometric coordinate functions, possessing the property of orthogonality. They enable us to determine eigenvalues not through the solution of the Sturm-Liouville boundary value problem, which supposes the second-order differential equation integration, but through the solution of a differential equation for an unknown function on time, which is the first-order equation. Due to the property of coordinate functions mentioned above, while determining constants of integration out of initial conditions it is possible to avoid solving large systems of algebraic linear equations with ill-conditioned matrix of coefficients. Thus, it simplifies both the process of obtaining a solution and its final formula and provides an opportunity to find not only an approximate, but also an exact analytical solution, represented by an infinite series.
\end{abstract}

Keywords: nonstationary heat conduction, L. V. Kantorovich method, Bubnov-Galerkin method, integral heat balance method, trigonometric coordinate functions, exact analytical solution, orthogonality of coordinate functions.

Received: $28^{\text {th }}$ October, $2016 /$ Revised: $11^{\text {th }}$ February, 2017 Accepted: $13^{\text {th }}$ March, $2017 /$ First online: $19^{\text {th }}$ March, 2017

\section{Short Communication}

(ㅇ)(i) The content is published under the terms of the Creative Commons Attribution 4.0 International License (http://creativecommons.org/licenses/by/4.0/)

Please cite this article in press as:

Kudinov V. A., Klebleev R. M., Kuklova E. A. Obtaining exact analytical solutions for nonstationary heat conduction problems using orthogonal methods, Vestn. Samar. Gos. Tekhn. Univ., Ser. Fiz.-Mat. Nauki [J. Samara State Tech. Univ., Ser. Phys. Math. Sci.], 2017, vol. 21, no. 1, pp. 197-206. doi:10.14498/vsgtu1521 (In Russian).

\section{Authors' Details:}

Vasiliy A. Kudinov (D) http://orcid.org/0000-0002-3071-5168

Dr. Phys. \& Math. Sci., Professor; Head of Dept.; Dept. of Theoretical Fundamentals of Heat-Engineering and Hydromechanics; e-mail: totig@yandex.ru

Ruslan M. Klebleev (D) http://orcid.org/0000-0002-6944-8503

Graduate Student; Dept. of Theoretical Fundamentals of Heat-Engineering and Hydromechanics; e-mail: uio1123@list.ru 
Competing interests. We have no competing interests.

Authors' contributions and responsibilities. Each author has participated in the article concept development and in the manuscript writing. The authors are absolutely responsible for submitting the final manuscript in print. Each author has approved the final version of manuscript.

Funding. This work was supported by the Russian Ministry of Education and Science within the base portion of the state task to Samara State Technical University (project no. 1.5551.2017/8.9).

\section{References}

1. Kantorovich L. V., Krylov V. I. Priblizhennye metody vysshego analiza [Approximate Methods of Higher Analysis]. Leningrad, Fizmatgiz, 1962, 708 pp. (In Russian)

2. Tsoi P. V. Sistemnye metody rascheta kraevykh zadach teplomassoperenosa [System methods for calculating the heat and mass transfer boundary value problems]. Moscow, Moscow Power Engineering Institute Publ., 2005, 568 pp. (In Russian)

3. Kudinov V. A., Kartashov E. M., Kalashnikov V. V. Analiticheskie resheniya zadach teplomassoperenosa i termouprugosti dlya mnogosloynykh konstruktsiy [Analytical problem solving heat and mass transfer and thermoelasticity for multilayer structures]. Moscow, Vysshaya shkola, 2005, 430 pp. (In Russian)

4. Kudinov V. A., Averin B. V., Stefaniuk E. V. Teploprovodnost' i termouprugost' $v$ mnogosloinykh konstruktsiiakh [Thermal Conductivity and Thermoelasticity in Multilayer Structures]. Moscow, Vysshaia shkola, 2008, 305 pp. (In Russian)

5. Lykov A. V. Teoriia teploprovodnosti [Theory of heat conduction]. Moscow, Vysshaia shkola, 1967, 600 pp. (In Russian) 